\title{
Fusion of Dominant Colour and Spatial Layout Features for Effective Image Retrieval of Coloured Logos and Trademarks
}

\author{
Yijun Yan ${ }^{1}$, Jinchang Ren ${ }^{1}$, Yinsheng Li $^{2}$, James Windmill ${ }^{1}$, Winifred Ijomah ${ }^{3}$ \\ ${ }^{1}$ Department of Electronic and Electrical Engineering, University of Strathclyde, Glasgow, UK \\ ${ }^{2}$ School of Computer Science, Fudan University, Shanghai, China \\ ${ }^{3}$ Dept. of Design, Manufacture and Engineering Management, Univ. of Strathclyde, Glasgow, UK
}

\begin{abstract}
Due to its uniqueness and high value in commercial side, logos and trademarks play a key role in e-business based global marketing. Detecting misused and faked logos need designated and accurate image processing and retrieval techniques. However, existing colour and shape based retrieval techniques, which are mainly designed for natural images, cannot provide effective retrieval of logo images. In this paper, an effective approach is proposed for content-based image retrieval of coloured logos and trademarks. By extracting the dominant colour from colour quantization and measuring the spatial similarity, fusion of colour and spatial layout features is achieved. The proposed approach has been tested on a database containing over 250 logo images. Experimental results show that the proposed methodology yields more accurate results in retrieving relevant images than conventional approaches even with added Gaussian and Salt\&pepper noise.
\end{abstract}

Keywords-Image retrieval; dominant colour descriptor; spatial layout; logo and trademark retrieval

\section{INTRODUCTION}

With the rapid development of e-business, the form and type of trademark/logo images have increased dramatically. However, the misuse and imitation of specified trademarks and logos also grows simultaneously, which have misled consumers and damaged their confidence in e-business. As a result, detection of such misusage becomes a research hotspot, where trademark/logo image retrieval (TLIR), as one subset of Content-based image retrieval (CBIR), is of great practical significance.

For identification of TLIR, two most important problems must be solved. One is how to extract applicable feature element to represent true colour logo image content, and the other is how to retrieve image effectively based on extracted feature vectors.

Colour, as one of the low-level visual features, is widely used in CBIR research. [1-3] It is one of the most important attributes for image matching and retrieval due to its invariance to image size and orientation. Several kinds of colour descriptors have been proposed, including colour moment $[4,5]$, colour histogram $[6,7]$, the colour coherence vector, colour correlogram $[8,9]$, dominant colour (DC), etc. For most chromatic logo images, the most salient characteristic is dominant colour which has two main components: (1) representative colour and (2) the percentage of each colour. Therefore the dominant colour descriptors are considered to be the most suitable feature extraction methods. In many current papers, dominant colour descriptor (DCD) is are very popular [10-13]. It is one of descriptors in MPEG-7, and it can describe the representative colour distributions in an image or a region of interest in an effective and compact form. For extraction of colour information, the selection of colour space is of great importance. Two kinds of colour space are widely used in DCD, one is RGB colour space and the other is HSV colour space. For RGB colour space, Yang et al. [12] presented a colour quantization method for dominant colour extraction, called the linear block algorithm (LBA). It has been shown that the LBA has high efficiency in colour quantization and computation. The combination of LBA and their modified distance measurement gave good similarity matching result which caters to human perception very well. For HSV colour space, H.Shao et al. [10] proposed an image retrieval system framework based on MPEG-7 DCD. It extracts representative colour information by using a noninterval quantization algorithm, and histogram intersection is applied to measure similarity. Experimental result proves that the method has a good performance.

Although DCD provides an efficient colour quantization and extraction way, it is really sensitive to the image quality. Since lots of logo images online don't have good resolution, so that the existing DCD-based retrieval methods can't give a pleasant result. Therefore, it is necessary to improve the colour quantization and find another feature descriptor to extract more information from the image. In this paper, K-means clustering is applied to optimize the colour quantization, and a spatial descriptor is added to get the global feature of the image.

Most researches [3] [14, 15] have done very good works on trademark and logo retrieval using shape descriptors, however, the drawback of shape descriptors is similar to colour descriptors, i.e. sensitive to the image quality. When the image is with noise or of bad resolution, its edges become fuzzy hence cannot be accurately detected and result in the failure of shape descriptors in such situations. However, spatial (layout) feature is different with shape feature, which is used to get the location and distribution of the objects. For low quality logo images, although their edges are blurred, the colour region is almost there, and spatial descriptor can extract these regions precisely. Meanwhile, the image database in most shape-based TLIR methods are grayscale images or manually images. In another word, their retrieval methods are not suitable for true colour logo image retrieval in the real world. Thus, spatial descriptor is used to obtain the spatial information of an image in this paper.

In this paper, color quantization, $\mathrm{K}$-means clustering and spatial feature extraction are combined for colour image retrieval of logos and trademarks. Experimental results have demonstrated that the integration of LBA and K-means clustering has better quantization performance than LBA. In addition, our proposed TLIR method is more robust to the 
Gaussian noise, which further validates the efficacy of our approach in this context.

The rest of paper is organized as follows. Section II describes the principle of the proposed method, which include extraction of colour and spatial layout features, and fusion based matching strategy. Section III presents the experimental results and performance analysis of our TLIR method evaluated on our collected logo database. Finally, a short conclusion is summarized in Section IV.

\section{PROPOSED METHOD}

\section{A. Color quantization}

In general, a true colour logo image has several salient colours. In order to extract these colours rapidly, dominant colour descriptor is considered as the most efficient method. LBA[12] is a very efficient colour quantization method and its computation cost is also very low. Given an image I, the RGB color space is uniformly divided into 8 coarse partitions as shown in Fig. 1.

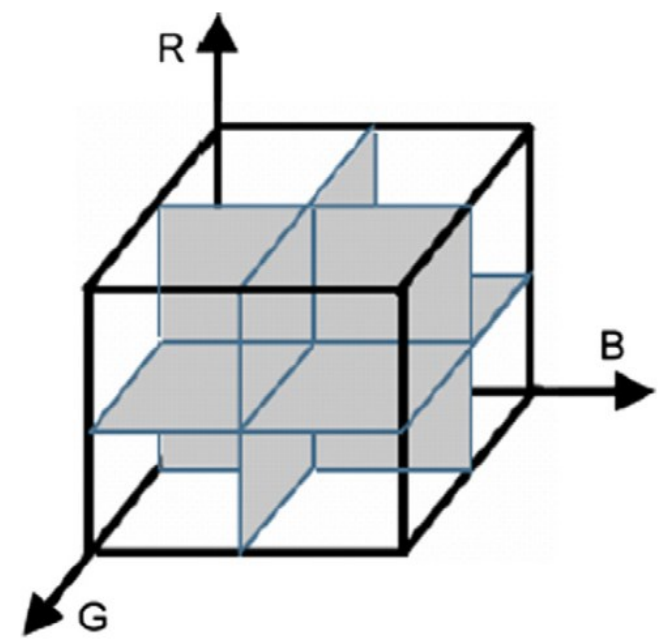

Fig. 1. The coarse partitions of RGB color space.

For each partition, the quantized color $C_{i}=\left(\bar{x}_{i}^{R}, \bar{x}_{i}^{G}, \bar{x}_{i}^{B}\right)$, $i \in[1,8]$. Where $\bar{x}_{i}$ is the average value of distribution of three color components red, green and blue, for each partition center. Afterwards, mutual distance of two adjacent $C_{i}$ is calculated and similar "colorbins" are merged together using weighted average agglomerative procedure:

$$
\left\{\begin{array}{l}
x^{R}=x_{1}^{R} \times\left(\frac{p_{R, 1}}{p_{R, 1}+p_{R, 2}}\right)+x_{2}^{R} \times\left(\frac{p_{R, 2}}{p_{R, 1}+p_{R, 2}}\right) \\
x^{G}=x_{1}^{G} \times\left(\frac{p_{G, 1}}{p_{G, 1}+p_{G, 2}}\right)+x_{2}^{G} \times\left(\frac{p_{G, 2}}{p_{G, 1}+p_{G, 2}}\right) \\
x^{B}=x_{1}^{B} \times\left(\frac{p_{B, 1}}{p_{B, 1}+p_{B, 2}}\right)+x_{2}^{B} \times\left(\frac{p_{B, 2}}{p_{B, 1}+p_{B, 2}}\right)
\end{array}\right.
$$

In Eq. (1), $p_{R}, p_{G}, p_{B}$ represent the percentage of R,G,B components, respectively. The merge processes iterate until the minimum Euclidian distance between the adjacent "colorbin" centre being larger than the threshold $T_{d}$. Since the dominant colors should be significant enough, if the percentage of survived colour is less than a threshold $T_{m}$, it will be merged into the nearest colour. In this study, we choose the $T_{d}$ as 25 and $T_{m}$ as $3 \%$.
Eventually, the LBA can quickly return maximum eight dominant colour descriptors (DCDs) and DCD in MPEG-7 is defined as $F_{C}=\left\{\left(C_{i}, P_{i}\right), i=1, \ldots M\right\}$. Here $C_{i}$ is the 3D dominant colour vectors, $P_{i}$ is the percentage for each dominant colour and the sum of $P_{i}$ equals $1, \mathrm{M}$ is the total number of dominant colour for an image. Fig. 2 shows the logo image of University of Strathclyde. In this image, there are five dominant colours and the quantization result generated by LBA exactly meets the human vision as shown in Table I.

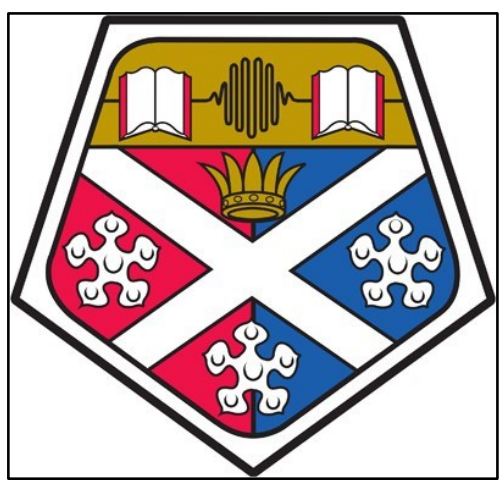

Fig. 2. The original logo image.

TABLE I

THE DOMINANT COLOUR FEATURE OF FIGURE2.

\begin{tabular}{lcccc}
\multicolumn{1}{c}{ Colour } & Red & Green & Blue & $\begin{array}{c}\text { Percentage } \\
(\%)\end{array}$ \\
\hline BLACK & 46 & 38 & 35 & 17.23 \\
RED & 221 & 26 & 71 & 8.1 \\
BLUE & 29 & 93 & 169 & 6.96 \\
YELLOW & 188 & 152 & 15 & 8.11 \\
WHITE & 251 & 251 & 251 & 59.61
\end{tabular}

However, if the image is with bad resolution or noise, the LBA can't give a proper quantization result as the noise affects both 3D dominant colour vectors and the percentage of each DCs. In order to improve the robust to image quality for colour quantization, the integration of LBA and K-means clustering algorithm is proposed. And L1 and L2 are used to compute the distance between centroid and the points in the clusters, where L1 distance is Manhattan distance and L2 distance is Euclidean distance.

From Fig. 3, it can be seen that the quantization results of integration of LBA and K-means on low quality image give better quantization performance on human vision. Peak signal to noise ratio (PSNR), and mean square error (MSE) are also used to evaluated the quantization performance. In general, if the value PSNR is higher and the value of MSE is lower, then the quantized image quality is better.

The MSE and PSNR between two images are given by:

$$
M S E=\frac{1}{N} \sum_{i=1}^{N}\left(x_{i}-y_{i}\right)^{2}
$$




$$
P S N R=10 \log _{10}\left(\frac{L^{2}}{M S E}\right)
$$

where $\mathrm{N}$ is the total number of pixels in the image and $\mathrm{L}$ is the maximum dynamic range. For 8 bpp images, $\mathrm{L}=255$. $x_{i}$ is quantized image and $y_{i}$ is reference image.

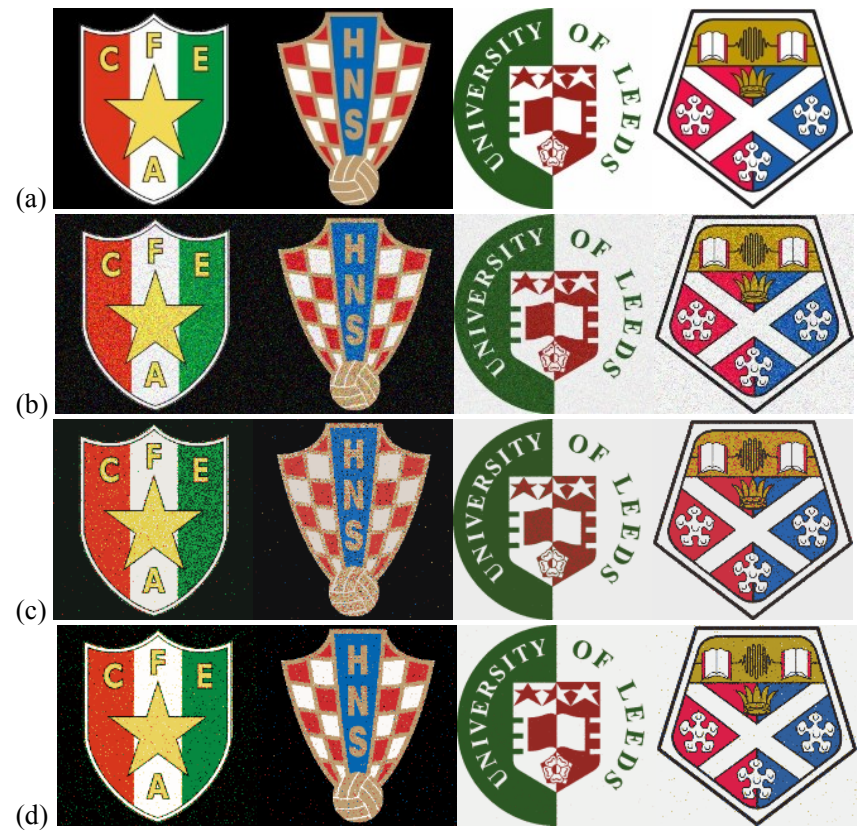

Fig. 3. (a) Original example logo image. (b) Logo image with Gaussian noise. (c) Quantization results from b using LBA method. (d) Quantization results from $b$ using integration of LBA and K-means.

TABLE II

MSE AND PSNR COMPARISON OF TWO QUANTIZATION METHODS.

\begin{tabular}{ccc|cc}
\hline Method & \multicolumn{2}{c}{ LBA } & \multicolumn{2}{c}{ LBA_Kmeans } \\
\hline $\begin{array}{c}\text { Gaussian } \\
\text { variance }\end{array}$ & $\begin{array}{c}\text { PSNR } \\
(\mathrm{db})\end{array}$ & MSE & $\begin{array}{c}\text { PSNR } \\
(\mathrm{dB})\end{array}$ & MSE \\
\hline $\mathbf{0 . 0 1}$ & 20.93 & 644.30 & 22.23 & 485.66 \\
$\mathbf{0 . 0 1 1}$ & 20.79 & 658.71 & 22.12 & 493.38 \\
$\mathbf{0 . 0 1 2}$ & 20.69 & 666.94 & 22.09 & 488.87 \\
$\mathbf{0 . 0 1 3}$ & 20.59 & 674.65 & 21.99 & 492.99 \\
$\mathbf{0 . 0 1 4}$ & 20.48 & 687.64 & 21.88 & 502.36 \\
$\mathbf{0 . 0 1 5}$ & 20.36 & 701.34 & 21.75 & 516.23 \\
$\mathbf{0 . 0 1 6}$ & 20.26 & 713.46 & 21.65 & 523.13 \\
$\mathbf{0 . 0 1 7}$ & 20.15 & 727.42 & 21.52 & 536.91 \\
$\mathbf{0 . 0 1 8}$ & 20.05 & 740.73 & 21.40 & 547.99 \\
$\mathbf{0 . 0 1 9}$ & 19.95 & 752.63 & 21.27 & 561.14 \\
\hline $\mathbf{0 . 0 2}$ & 19.83 & 770.19 & 21.14 & 577.16 \\
\hline $\mathbf{0 . 0 2 1}$ & 19.77 & 778.38 & 21.05 & 584.30 \\
\hline $\mathbf{0 . 0 2 2}$ & 19.63 & 798.84 & 20.90 & 603.17 \\
$\mathbf{0 . 0 2 3}$ & 19.54 & 812.97 & 20.79 & 613.57 \\
\hline $\mathbf{0 . 0 2 4}$ & 19.45 & 826.62 & 20.68 & 627.05 \\
$\mathbf{0 . 0 2 5}$ & 19.34 & 844.03 & 20.54 & 642.97 \\
\hline & & & & \\
\hline
\end{tabular}

Table II shows the average PSNR and MSE value with several Gaussian variance level evaluated on our logo image database. It proves that KLBA (the combination of LBA and Kmeans) gives better quantization results that the original LBA approach.

\section{B. Colour dissimilarity}

The DCD similarity matching strategy is based on Yang [2]. The distance between two images $I_{1}$ and $I_{2}$ is defined as:

$$
\text { Dis_C }\left(I_{1}, I_{2}\right)=1-\operatorname{SIM}\left(I_{1}, I_{2}\right)
$$

And the similarity measure is defined as: $\left(I_{1}, I_{2}\right)=$ $\sum_{i=1}^{N_{1}} \sum_{j=1}^{N_{2}} a_{i, j} S_{i, j}$, and similarity score between two different representative colours is defined as:

$$
S_{i, j}=\left[1-\left|p_{q}(i)-p_{t}(j)\right|\right] * \min \left(p_{q}(i), p_{t}(j)\right.
$$

where $p_{q}(i)$ and $p_{t}(j)$ are the percentage of the $i$ th and $j$ th dominant color in query image and target image, respectively. The term in bracket, $1-\left|p_{q}(i)-p_{t}(j)\right|$, is used to measure the difference of two colors in their percentage. The $\min \left(p_{q}(i), p_{t}(j)\right)$ is the intersection of $p_{q}(i)$ and $p_{t}(j)$, which represents the similarity between two colors in percentage. If $p_{q}(i)$ equals to $p_{t}(j)$, then their percentage is same and the color similarity is determined by $\min \left(p_{q}(i), p_{t}(j)\right)$, otherwise, a large difference between $p_{q}(i)$ and $p_{t}(j)$ will decrease the similarity measure.

The similarity coefficient is given by:

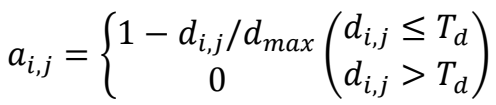

$$
\begin{aligned}
& d_{i, j}=\left\|c_{i}-b_{j}\right\|
\end{aligned}
$$

$d_{i, j}$ is Euclidean distance between two colour clusters $c_{i}$ and $b_{j} . T_{d}$ is the threshold to judge if two colour feature are similar, and $d_{\max }=\alpha T_{d}$. The parameter $\alpha$ is set to 2 and $T_{d}$ is 25 in this work.

\section{Spatial descriptor}

The spatial location in an image represents the spatial distribution information of the colors in the image. M.S.Kankanhallo [17] has proposed a useful spatial descriptor and the steps of spatial information extraction list as follows:

i. Split image into several layers and the number of these layers equals the number of dominant color.

ii. Label connected components to generate the spatial clusters in each colour layer.

iii. Define lower threshold is $\mathrm{P}^{*} 0.03125 \%$, upper threshold is $\mathrm{P} * 0.625 \%$ ( $\mathrm{P}$ is the total pixels in an image) and density function is given by: $=\frac{N}{l_{\max }^{2}}$, where $\mathrm{N}$ is the population of the cluster. Assume points $\left(x_{1}, y_{1}\right)$ and $\left(x_{2}, y_{2}\right)$ are the corner points of a rectangle which bounds a particular spatial cluster. So $l_{\max }$ is the maximum length of box, which is defined as $l_{\max }=$ $\max \left(\left\|x_{1}-x_{2}\right\|,\left\|y_{1}-y_{2}\right\|\right) \quad[16]$.

iv. Discard the clusters of which the population is less than the lower threshold. For the clusters which have population less than the upper threshold but more than lower threshold, eliminate those clusters which density $\rho$ is less than $10 \%$. 

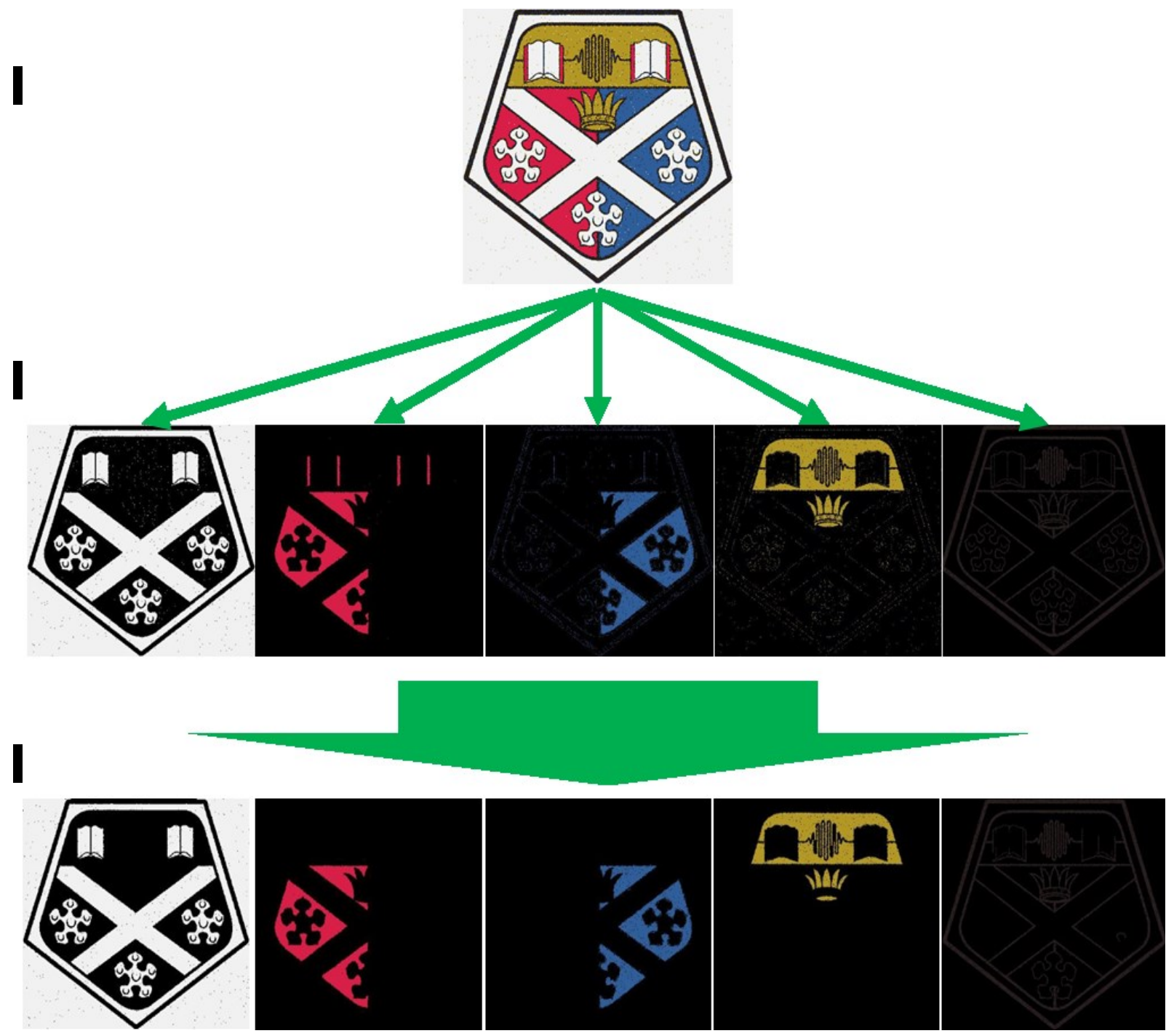

Fig. 4. Quantized logo image (top); result of image segmentation (middle); and result of population and density filter (bottom).

v. Find the centroid location from survived spatial clusters.

Then the colour-spatial feature vector is defined as:

$$
\mathrm{V}=\left\{C_{i}, P_{i}, x_{i j}, y_{i j}\right\},(i \in[1, M], j \in[1, N])
$$

where $C_{i}$ and $P_{i}$ are the dominant colour and their corresponding percentage respectively. $\left(x_{i j}, y_{i j}\right)$ is the centroid of the $j$ th spatial cluster in $i$ th colour layer. $\mathrm{M}$ is the number of DC, and $N$ is the number of spatial cluster in certain colour layer.

Fig. 4 shows the sample result of spatial clustering. It can be seen that few spatial clusters are inevitably lost, but the main spatial information is extracted in each colour layers.

\section{Spatial dissimilarity}

Assume the query image $I_{q}$ has i spatial clusters in $m^{\text {th }}$ dominant colour layer and target image $I_{t}$ has j spatial clusters in nth dominant colour layers. If $\mathrm{mth}$ dominant colour in $I_{q}$ is similar to nth dominant colour in $I_{t}$, the assignment function $\mathrm{P}$ maps every spatial cluster $s$ in $I_{q}$ to the closest color cluster $\mathrm{P}_{s}$ in $I_{t}$.This is a 1-to-1 mapping and $\mathrm{P}$ is determined as follows. i. Form the distance matrix, $D_{i j}$

$$
D_{i j}=\left[d_{i j}\right]_{i * j}
$$

ii. Find the minimum entry $(a, b)$ in matrix $D_{i j}$ and note that $\mathrm{P}(\mathrm{a})=\mathrm{b}$,

iii. Cut off the row a and column b of the matrix $D_{i j}$. If the matrix $D_{i j}$ is non-degenerate, go to step 2 else stop. A matrix is considered degenerate when the number of rows or columns which is not yet struck off is zero.

For every matched spatial cluster, using the spatial centroid for distance measure,

$$
\begin{gathered}
d=\frac{\sum_{k=1}^{\min (i, j)} \sqrt{\left(x_{k}^{Q}-x_{P_{S}(k)}^{T}\right)^{2}+\left(y_{k}^{Q}-y_{P_{S}(k)}^{T}\right)^{2}}}{\min (i, j)} \\
\text { Dis_S }=\frac{1}{M} \sum_{m=1}^{M} d(m)
\end{gathered}
$$

where $\mathrm{M}$ is the number of similar colour. 


\section{E. Fusion of Color and Spatial Features}

In general, the proposed fusion of color and spatial feature extraction method is similar to several object detection tasks [17-20]. In the proposed retrieval system, however, it first decides whether two images have similar colour. If not, this pair of images is considered as different. Otherwise, the system measures spatial feature difference for every similar color layers. And global dissimilarity is defined by

$$
D=\gamma \cdot * D i s_{-} S+(1-\gamma) . * D i s_{-} C
$$

where $\gamma$ is used to weight colour and spatial based features when determining the similarity of the image.

\section{EXPERIMENTAL RESULTS}

To validate the efficacy of the proposed approach, we have collected our own database for performance assessment. The experimental settings and detailed comparison of experimental results are reported as follows.

\section{A. Experiment setup}

To evaluate the performance of trademark retrieval system, database is one of the significant issues. Although MPEG-7 is popular in some trademark retrieval research[21, 22], the images are not deliberately designed for performance evaluation of colour logo image retrieval system. Therefore, in this project, we build our own colour logo image database, which contains 266 original images and a number of distorted versions. The original images were collected from online and include 135 Britain university logo images, 50 football team logo images and some vector diagrams, where some of them are very similar to each other. The whole database will make public in the future. Moreover, live feedback can also be added to allow users' interaction with the system [23].

For each of the 266 logo/trademark images, noise was added to generate degraded versions for performance assessment. The distortion types include 16 levels of Gaussian noise and 4 levels of salt \& pepper noise. The logo images with noises are considered as query image, the target image is source image. A single value measurement, average precision $\left(P_{\text {ave }}\right)$, is used to estimate the global performance of the retrieval systems. For a query image, if the system can retrieve its ground truth image in a number of retrieval results, $\mathrm{P}$ is 1 , otherwise 0 .

$$
P_{\text {ave }}(R)=\frac{1}{N q} \sum_{i=1}^{N q} P_{i}(R)
$$

where $\mathrm{Nq}$ is the number of query images, $\mathrm{R}$ is the number of top retrieval result and $\mathrm{R}$ is set as 1 and 5 .

\section{B. The comparison average precision}

To evaluate the retrieval performance, several retrieval schemes are compared with.

i. Colour retrieval method (LBA) proposed by Yang [12].

ii. Integration of LBA and spatial feature with global dissimilarity measure.

iii. Colour retrieval method (HSV_DCD) proposed by Shao[10].

iv. Integration of LBA and K-means (L1 distance) with colour dissimilarity measure.

v. Integration of LBA and K-means (L2 distance) with colour dissimilarity measure.

vi. Integration of LBA, K-means (L1) and spatial feature with global dissimilarity measure.

vii. Integration of LBA, K-means (L2) and spatial feature with global dissimilarity measure.

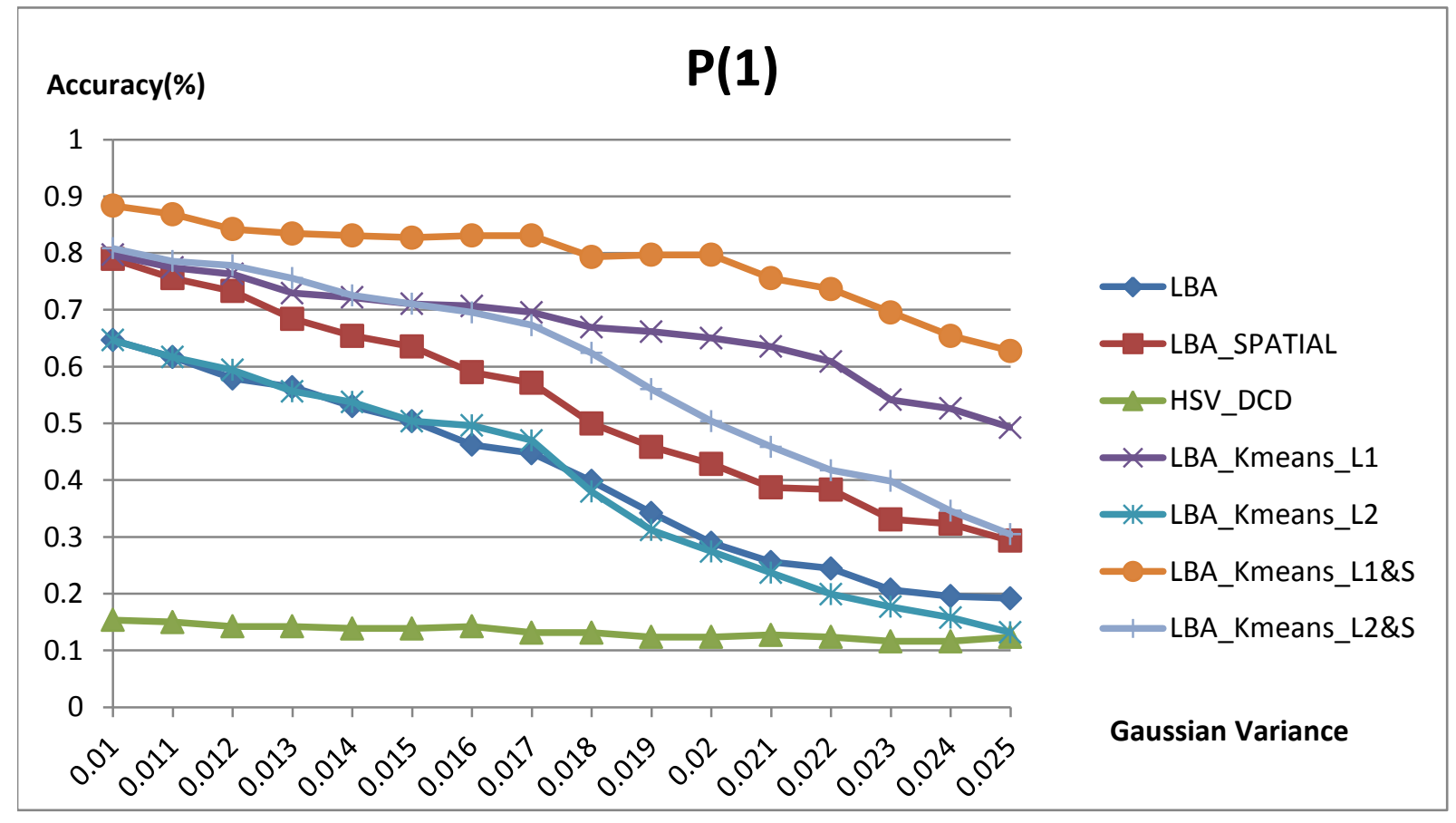

Fig. 5. Performance comparisons of different retrieval strategies over Gaussian noise dataset when $\mathrm{R}$ is 1 . 


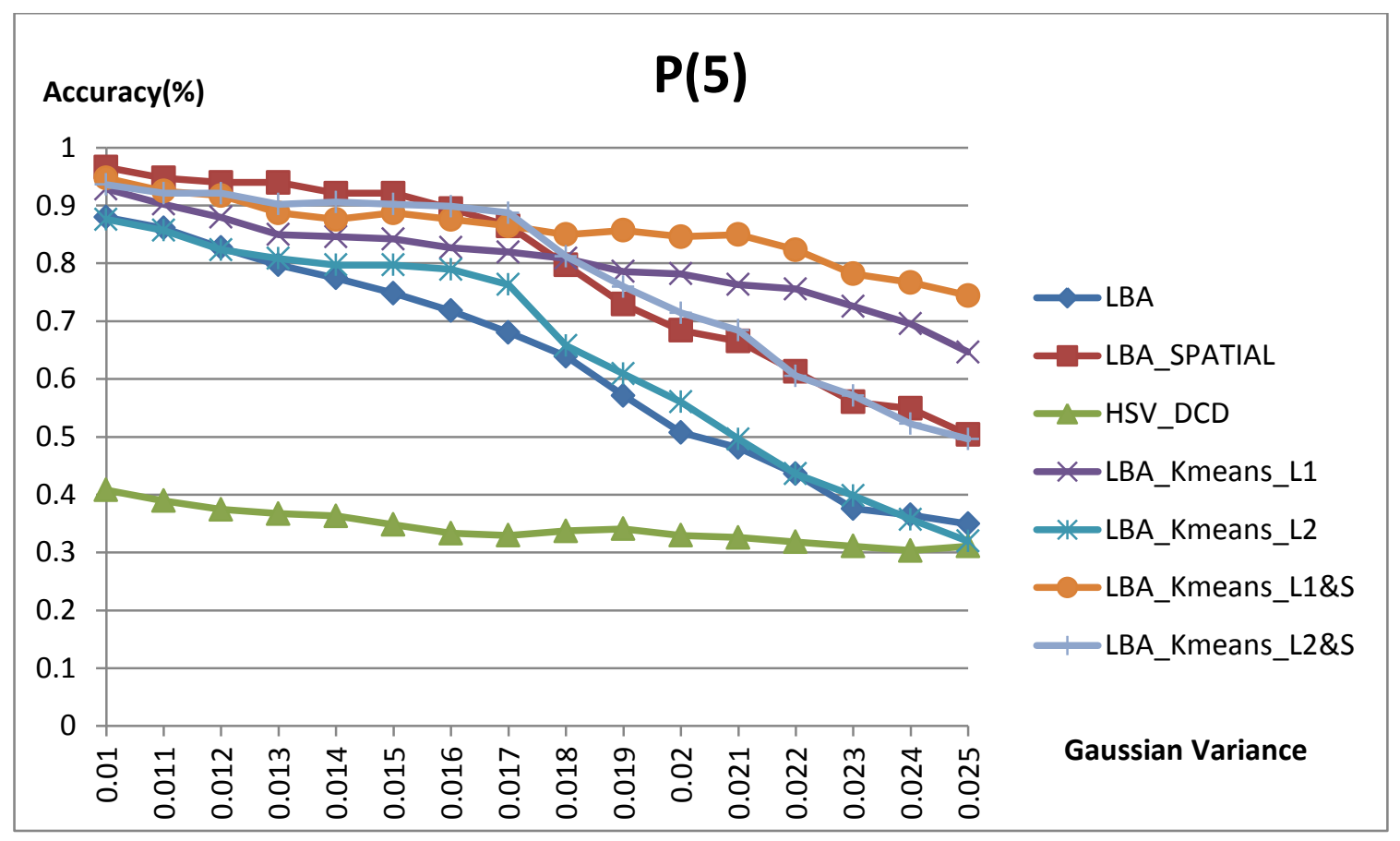

Fig. 6. Performance comparisons of different retrieval strategies over Gaussian noise dataset when R is 5 .

Fig. 5 and Fig. 6 show performance comparisons of different retrieval strategies over Gaussian noise dataset. It is obviously that HSV_DCD has worst performance. This is because the HSV-based method quantize the whole image into $(8 * 3 * 3=72)$ colour bins, where too many bins have inevitably enlarged the effect of noisy samples and result in unreliable colour quantization and poor retrieval results. Unlike HSVbased method, the LBA method is based on RGB colour space and it quantize the true colour image into maximum 8 representative colours, which not sensitive to the noise a lot. And the integration of LBA and K-means get better performance than LBA in retrieval results, especially when Kmeans clustering use L1 distance. On the other hand, we can also see that, after combining both colour and spatial feature and using adaptive coefficient, the average precision is higher than the method with only colour feature. In Fig. 5, the integration of LBA and spatial method outperforms all other method when the noise is not strong. However, with the raise of the noise, its performance goes worse, but the integration of KLBA and spatial method display its robustness to the noise increasingly and gives the best overall performance. Meanwhile, the method which includes K-means clustering with L1 distance has better performance than that with L2 distance since L1-distance is known to be robust to noise [24, 25]. Therefore, our proposed method can be considered robust to low quality trademark/logo image.

For Salt and Pepper noise, Tables III-IV show the performance of the retrieval results using $\mathrm{P}(1)$ and $\mathrm{P}(5)$, respectively. As HSV_DCD produces the worst results, it is excluded in our experiments and results in 6 groups of results given for comparison. Again, we can find that the proposed approach which combines LBA, K-means (L1) and spatial layout features consistently yield the best results again under different levels of Salt and Pepper noise. With the fusion of spatial feature, the $\mathrm{P}(1)$ and $\mathrm{P}(5)$ accuracy is actually improved by $14 \%$ and $7 \%$, respectively.
TABLE III

P(1) PERFORMANCE COMPARISONS OF DIFFERENT RETRIEVAL STRATEGIES WITH SALT\&PEPPER NOISE.

\begin{tabular}{|c|c|c|c|c|}
\hline Method Noise density & 0.05 & 0.1 & 0.15 & 0.2 \\
\hline LBA & 0.6466 & 0.3459 & 0.1992 & 0.1429 \\
\hline LBA+Spatial & 0.7406 & 0.4436 & 0.2632 & 0.218 \\
\hline KLBA_L1 & 0.7368 & 0.5752 & 0.4323 & 0.2932 \\
\hline KLBA_L2 & 0.6429 & 0.5301 & 0.3797 & 0.2105 \\
\hline KLBA\& SPATIAL_L1 & 0.8759 & 0.7744 & 0.6053 & 0.4688 \\
\hline KLBA\& SPATIAL_L2 & 0.7669 & 0.4662 & 0.3684 & 0.297 \\
\hline
\end{tabular}

TABLE IV

P(5) PERFORMANCE COMPARISONS OF DIFFERENT RETRIEVAL STRATEGIES WITH SALT\&PEPPER NOISE.

\begin{tabular}{|c|c|c|c|c|}
\hline Method Noise density & 0.05 & 0.1 & 0.15 & 0.2 \\
\hline LBA & 0.8459 & 0.5752 & 0.391 & 0.282 \\
\hline LBA+Spatial & 0.9023 & 0.6541 & 0.4586 & 0.3684 \\
\hline KLBA_L1 & 0.8684 & 0.8008 & 0.688 & 0.5 \\
\hline KLBA_L2 & 0.8008 & 0.7068 & 0.5564 & 0.4098 \\
\hline KLBA\& SPATIAL_L1 & 0.9323 & 0.8759 & 0.7594 & 0.6805 \\
\hline KLBA\& SPATIAL_L2 & 0.8684 & 0.6579 & 0.5338 & 0.4737 \\
\hline
\end{tabular}

\section{CONCLUSION}

In this paper, a novel combined approach is proposed for effective content-based retrieval of colour logo/trademark images. By fusion of dominant colour extraction (using colour quantization and $\mathrm{K}$-means clustering) and spatial layout 
features, the retrieval accuracy measured by $\mathrm{P}(1)$ and $\mathrm{P}(5)$ has been significantly improved. Even with Gaussian and Salt \& Pepper noise added, the proposed approach can still yield very satisfied results. In addition, it is found that Manhattan distance produces much better results that Euclidean distance. Future work includes applying the proposed approach to other datasets as well as adaptively parameter determination in the proposed approach to balance between colour and spatial layout features.

\section{ACKNOWLEDGEMENTS}

The authors would like to thank the partial support of the EU GreeNet project that connects us together for this interesting topic.

\section{REFERENCE}

[1] T.-C. Lu, and C.-C. Chang, "Color image retrieval technique based on color features and image bitmap," Information Processing \& Management, vol. 43, no. 2, pp. 461-472, 2007.

[2] C.-H. Lin, R.-T. Chen, and Y.-K. Chan, "A smart content-based image retrieval system based on color and texture feature," Image and Vision Computing, vol. 27, no. 6, pp. 658-665, 2009.

[3] X.-Y. Wang, Y.-J. Yu, and H.-Y. Yang, "An effective image retrieval scheme using color, texture and shape features," Computer Standards \& Interfaces, vol. 33, no. 1, pp. 59-68, 2011.

[4] Z. Huang, "CONTENT-BASED IMAGE RETRIEVAL USING COLOR MOMENT AND Gabor Texture Feature," in Machine Learning and Cybernetics, Qingdao, 2010, pp. 719-724.

[5] P. Maheshwary, and N. Srivastav, "Retrieving Similar Image Using Color Moment Feature Detector and K-Means Clustering of Remote Sensing Images," pp. 821-824, 2008.

[6] G.-H. Liu, and J.-Y. Yang, "Content-based image retrieval using color difference histogram," Pattern Recognition, vol. 46, no. 1, pp. 188-198, 2013.

[7] S. Jeong, C. S. Won, and R. M. Gray, "Image retrieval using color histograms generated by Gauss mixture vector quantization," Computer Vision and Image Understanding, vol. 94, no. 1-3, pp. 44-66, 2004.

[8] T. T. U. Leauhatong, Hiratsuka ; Atsuta, K. ; Kondo, S., "A New Contentbased Image Retrieval Using Color Correlogram and Inner Product Metric," in Image Analysis for Multimedia Interactiive Service, Santorini, 2007, pp. 33.

[9] G. Y. A. I. J. G. K. Jongan Park ; Chosun Univ., "Image Indexing using Spatial Multi-Resolution Color Correlogram," in Imaging Systems and Techniques, Krakow, 2007, pp. 1 - 4.

[10] H. Shao, Y. Wu, W. Cui et al., "Image Retrieval Based on MPEG-7 Dominant Color Descriptor," in Young Computer Scientists, Hunan, 2008, pp. 753-757.
[11] R. Min, and H. D. Cheng, "Effective image retrieval using dominant color descriptor and fuzzy support vector machine," Pattern Recognition, vol. 42, no. 1, pp. 147-157, 2009.

[12] N.-C. Yang, W.-H. Chang, C.-M. Kuo et al., "A fast MPEG-7 dominant color extraction with new similarity measure for image retrieval," J. Visual Communication and Image Representation, vol. 19, no. 2, pp. 92-105, 2008.

[13] A. Talib, M. Mahmuddin, H. Husni et al., "A weighted dominant color descriptor for content-based image retrieval," J. Visual Communication and Image Representation, vol. 24, no. 3, pp. 345-360, 2013.

[14] M. Jian, and L. Xu, "Trademark Image Retrieval Using Wavelet-based Shape Features," in Intelligent Information Technology Application Workshops, Shanghai, 2008, pp. 496-500.

[15] Y.-j. Wang, and C.-f. Zheng, "Trademark Image Retrieval Based on Shape and Key Local Color Features," in Information and Computing Science, Manchester, 2009, pp. 325-328.

[16] B. M. M. Mohan S. Kankanhalli, Hock Yiung Huang, "Color and spatial feature for content-based image retrieval," Pattern Recognition Letters, vol. 20, no. 1, pp. 109-118, 1999.

[17] Y. Feng, J. Ren, and J. Jiang, "Object based 2D-to-3D video conversion for effective stereoscopic content generation in 3D-TV applications," Broadcasting, IEEE Transactions on, vol. 57, no. 2, pp. 500, June, 2011.

[18] J. Han, S. He, X. Qian et al., "An object_oriented visual saliency detection framework based on sparse coding representations," IEEE Trans. on Circuits and Systems on Video Technology, vol. 23, no. 2, pp. 2009-2021, Dec., 2013.

[19] J. Han, D. Zhang, G. Cheng et al., "Object detection in optical remote sensing images based on weakly supervised learning and high-level feature learning," IEEE Trans. Geoscience Remote Sensing, 2015, to appear.

[20] J. Han, K. N. Ngan, M. Li et al., "Unsupervised extraction of visual attention objects in color images," IEEE Trans. on Circuits and Systems on Video Technology, vol. 16, no. 1, pp. 141-145, January, 2006.

[21] H. Qi, K. Li, Y. Shen et al., "An effective solution for trademark image retrieval by combining shape description and feature matching," Pattern Recognition, vol. 43, no. 6, pp. 2017-2027, 2010.

[22] F. Mohd Anuar, R. Setchi, and Y.-k. Lai, "Trademark image retrieval using an integrated shape descriptor," Expert Systems with Applications, vol. 40, no. 1, pp. 105-121, 2013.

[23] J. Jiang, J. Kohler, C. M. Williams et al., "LIVE: an integrated production and feedback system for intelligent and interactive TV broadcasting," IEEE Trans. Broadcasting, vol. 57, no. 3, pp. 646-661, 2011.

[24] T. Mathew, "Least squares and least absolute deviation procedures in approximately linear models," Statistics \& Probability Letters, vol. 16, no. 2, pp. 153-158, 1993.

[25] H. R. M.F. Calitz, "Least absolute deviation (LAD) image matching," ISPRS Journal of Photogrammetry and Remote Sensing, vol. 51, no. 5, pp. 223-229, 1996. 